\title{
Research Progress and Review of Insomnia in Traditional Chinese Medicine
}

\author{
Jing Wang ${ }^{1}$, Xiufeng Fan ${ }^{2, *}$, Liqin $\mathrm{Zhi}^{2}$ \\ ${ }^{1}$ Shaanxi University of Traditional Chinese Medicine, Xianyang 712046, Shaanxi, China. \\ ${ }^{2}$ Department of Cardiology, Fifth Hospital, Xi'an, Shaanxi Province, Xi'an 710082, China.
}

How to cite this paper: Jing Wang, Xiufeng Fan, Liqin Zhi. (2020) Research Progress and Review of Insomnia in Traditional Chinese Medicine. International Journal of Clinical and Experimental Medicine Research, 4(4), 228-235.

DOI: 10.26855/ijcemr.2020.10.018

Received: October 15, 2020

Accepted: November 8, 2020

Published: November 16, 2020

*Corresponding author: Xiufeng Fan, Department of Cardiology, Fifth Hospital, Xi'an, Shaanxi Province, Xi'an 710082, China.

Email: 1092001897@qq.com

The first author: Jing Wang, Master candidate, Class of 2018, Shaanxi University of Traditional Chinese Medicine, Xianyang, Shaanxi 712046, China.

\begin{abstract}
This paper discusses the name, etiology and pathogenesis of insomnia, the thinking and treatment of insomnia in TCM, summarizes the clinical efficacy and advantages of traditional Chinese medicine in the treatment of insomnia, and puts forward the new ideas and methods of Shen's female department in the treatment of insomnia, so as to provide reference for scientific research and clinical treatment of insomnia in Chinese medicine.
\end{abstract}

\section{Keywords}

Insomnia, Syndrome differentiation and treatment, TCM treatment, Shen's Gynecology, Research progress

With the accelerated pace of modern life, cardiovascular disease, hypertension, anxiety and other mental disorders have a higher impact on human health. At present, Chinese and Western medicine treatment of insomnia have a certain effect, western medicine treatment of insomnia drugs are generally divided into three categories, the first type for benzodiazepines, such as diazepam, alprazolam; the second type is barbiturates, such as phenobarbital; the third is diphenylmethane, such as antalyl [1]. Western medicine has a certain therapeutic effect on insomnia, but at the same time, it will produce a lot of dependence and adverse reactions after withdrawal. Traditional Chinese medicine is based on different syndrome types and patient constitution through syndrome differentiation and treatment, so TCM treatment of insomnia shows its unique advantages [2].

Insomnia belongs to the category of "insomnia" in traditional Chinese medicine. It is a kind of sleep disorder syndrome which cannot sleep normally and seriously affect work and life. The main clinical manifestations are poor sleep quality, such as difficulty in falling asleep, difficulty in sleeping again after waking up or waking up from time to time, and even unable to fall asleep day and night [3]. There are different records about the name, etiology, pathogenesis and treatment methods of insomnia in the past dynasties. Through the literature review of insomnia, the author hopes to analyze the etiology, pathogenesis and treatment principles of insomnia from different dimensions. 


\section{Study on the name of insomnia}

\subsection{From pre Qin to the Three Kingdoms period}

In ancient medical books, the earliest names for insomnia include "not lying”, "not lying” and "not lying”. For example, the 120,000 character silk book "shiyimai Jiujing" unearthed from tomb No. 3 of Mawangdui said: do not eat, do not lie If you can't lie down, you're upset... If you can't lie down, you'll be forced to owe “Ten questions” [5]: "a night does not lie, a hundred days no longer". Such names were named as restless, unable to lie, unable to sleep, unable to sleep, unable to sleep in the canon of internal medicine, as well as less lying, unable to lie, etc. there are 25 places named "lying", and the name "no lying" is particularly common in this place, with a total of 15 places [6]. In addition, when expounding the etiology and treatment of this kind of disease, there are many names of insomnia that can't lie, can't sleep and can't sleep in “Treatise on Febrile Diseases” and "synopsis of Golden Chamber" written by Zhang Zhongjing of Han Dynasty, such as treatise on febrile diseases, differentiation of pulse and syndrome of Shaoyin disease and treatment [7]: "Shaoyin disease, which can last for more than two or three days, cannot lie in the heart, and Huanglian Ejiao decoction is the main one. Synopsis of golden chamber treatment of blood Bi deficiency and fatigue disease and pulse syndrome” [8] says: "deficiency of labor, deficiency of trouble, cannot sleep, Suanzaoren Decoction main". "Synopsis of the Golden Chamber-jaundice disease": "abdominal fullness, tongue flaccidity yellow, restlessness cannot sleep, belongs to Huang family”.

\subsection{Wei Jin Sui Tang period}

In Jin Dynasty, Wang Shuhe [9] described insomnia as "not sleeping”, "not lying”, “not sleeping”, "lying restless". The name of "insomnia" was recorded for the first time in the etiology and pathogenesis of insomnia in "waitai secret Yao · Volume 3" written by Wang Tao of Tang Dynasty The practice of doctor's diagnosis begins with the strong color of the neck, followed by insomnia and fever, in the irritable thinking of water, and finally produces sores and diarrhea, which are all in this ear" [10]. After the Jin and Tang Dynasties, the poor sleep quality was described, and the name "no sleep" was often used [11].

\subsection{Song, Jin and Yuan Dynasties}

"Do not sleep" and "do not sleep" are used more in the ancient medical literature of Song Dynasty. For example, in the treatise on the etiology and pathogenesis of insomnia in Song Dynasty, Chen Yan said: "treat gallbladder deficiency and cold, vertigo, flaccid feet,... Do not sleep” [12]. Most of the Jin and Yuan Dynasties called insomnia "no sleeping”, "no sleeping” [13].

\subsection{Ming and Qing Dynasties}

In the Ming and Qing Dynasties, the most commonly used description of insomnia was still "no sleeping”, "no sleeping" [14]. In Ming Dynasty, the first work that put forward insomnia to do special research and discussion was Dai Yuanli’s Secret conversion of syndrome differentiation and treatment tips [15].

\subsection{Modern times}

The term "insomnia", which is more commonly used in modern times, is not used much in ancient times. The name of insomnia in ancient times is "no sleep", which is often used to describe the symptoms of poor sleep during fever, but not the name of disease. It was not widely used by doctors until the Ming and Qing Dynasties, especially in modern times [16]. In 1997, the national standard of the people's Republic of China, terms of clinical diagnosis and treatment of traditional Chinese medicine, part of diseases, which was approved and promulgated by the State Administration of technical supervision, named the disease "insomnia" as "insomnia" and currently "insomnia" is used as the legal name of traditional Chinese medicine disease [17].

To sum up, from the historical evolution of TCM disease names of insomnia, the ancient medical literature mostly used "eyes not closed”, “can’t sleep” and “can’t sleep” to record the name of insomnia. After modern times, "insomnia" and "insomnia" are often used [18].

\section{Etiology and pathogenesis of insomnia}

Normal sleep plays an important role in the growth and development of the body and the regulation of various nerve functions. The Qi and blood of Zang Fu organs are sufficient and harmonious, the heart is stable, and the Wei Yang enters into Ying Yin. The stable state of yin and Yang is the basis of normal sleep. On the contrary, if exces- 
sive thinking hurts the spirit and spleen, or is scared to cause heart, gallbladder and Qi deficiency, or improper diet hurts stomach, stomach disharmony leads to insomnia, or old body deficiency, yin deficiency and hyperactivity of fire cause insomnia [19]. In a word, insomnia is mainly caused by emotional disorders, improper diet, work and leisure disorders, and imbalance of body deficiency and viscera after the disease. The disease location is in the heart, spleen, liver, gallbladder and kidney. The pathogenesis of insomnia is the disharmony of yin and Yang, the disharmony of yin and Yang, the incompatibility of yin and Yang, the prosperity of Yang and the decline of Yin.

\subsection{Emotional disorder}

Emotional disorder is closely related to liver dysfunction. Liver qi can smooth Qi, regulate emotion, promote initiative, hurt liver by anger, and disturb the mind by liver fire [20]. For example, according to the theory of blood syndrome [21], the liver contains the soul if the Yang is floating outside, the soul is not as good as the liver, then you can't sleep. Based on the above understanding, Wang qiechu [22] put forward the basic pathogenesis of insomnia through research, that is, the imbalance of liver yin and Yang, and the disharmony of Qi and blood. Yuan yunshuo [23] used Longdan Xiegan Decoction to treat insomnia with liver fire disturbing heart syndrome. Conclusion: liver plays an important role in regulating human sleep.

\subsection{Improper diet}

The spleen and stomach are the hinge of the human body's qi movement. Improper diet, excessive drinking and overeating, excessive eating of five flavors or eating too much fat and thick taste can damage the stomach, block Qi, brew phlegm heat, lead to disharmony of stomach qi, and even cause dysfunction of large intestine transportation and causing insomnia [24]. For example, in Su Wen, the theory of inverse tune [25], it is said that "a man cannot lie down if he has adverse Qi. It's the reverse of Yangming Yang Ming is the stomach pulse, and the stomach is the sea of six Fu organs. Its Qi also goes down. Yang Ming can’t follow its way, so it can’t lie down”. Xiajing [26] said: "stomach disharmony, lying uneasy. This is also called Therefore, improper diet, unfavorable spleen and stomach axis, resulting in the abnormal operation of Ying and Wei are the basis of insomnia".

\subsection{Maladjustment of overwork}

Excessive relaxation and overwork can damage the spleen and cause Spleen Qi weakness. The physiological function of the spleen is mainly to transport, promote the clearing and control the blood. The spleen is the source of Qi and blood biochemistry [27]. Spleen deficiency and loss of circulation will lead to deficiency of Qi and blood, resulting in insufficiency of heart blood, which cannot be served on the heart, and loss of mind and spirit can cause insomnia [28]. For example, Lin Peiqin, a famous doctor in Qing Dynasty, wrote "insomnia due to syndrome classification" [29]: "the spleen is injured by thinking, and the spleen is deficient in blood and cannot sleep for years". Therefore, overwork is a prerequisite for insomnia.

\subsection{After the disease, the body is deficient and the Zang Fu organs are out of balance}

Long illness or old age, deficiency of body and blood, resulting in lack of heart blood, blood does not nourish the spirit, heart loss, cause insomnia, or high Yin and yang deficiency leads to insomnia [30]. In addition, sun Qingchun [31] believes that the deficiency of Yin in the body, combined with excessive room work, kidney Yin loss, Yang failure and lower, can't help the heart, water and fire are not conducive, heart and kidney loss is also a major factor of insomnia. Volume 21 [32] of xumingyilei case clearly records the close relationship between people's sleep and the five zang organs of heart, liver, spleen, lung and kidney.

\subsection{Others}

In addition to the above understanding, Liu Hejian [33] of the Jin and Yuan Dynasties argued on the theory of fire and heat. He believed that somniloquy and dreaminess were internal heat, and realized that fire and heat disturbing the heart could lead to insomnia. However, Li Dongyuan especially emphasized the relationship between spleen and stomach and vitality. In his treatise on spleen and stomach [34], he put forward the relationship between sleep and bedding diet, that is, "if you don't sleep well at night, your bed is thick and hot. Therefore, if you go to it in a hurry, you should still try to sweat. If you are thin and uneasy, you can sleep naturally. If you are hungry and sleepy, you should eat less; if you are full and sleep uneasy, you should sit less”. 


\section{Thinking of diagnosis and treatment of insomnia in TCM}

\subsection{Adjust sleep time}

The basic starting point of traditional Chinese medicine treatment of insomnia is not the rapid change of forced abnormal sleep state, but the recovery of normal sleep and awakening as far as possible. That is, the rhythm of time should be observed to adjust sleep [35]. According to the changes of the four seasons in a year, ancient Chinese health preserving experts put forward the sleep time of different seasons [36], that is, the sleep time in spring, which is "sleeping at night and getting up early". That is to say, sleep at night and get up at dawn. The sleep time in summer is "sleep in the night and get up early, so don't be tired of the day". "In summer and March, it's better to go to bed late and get up early, so as to feel the fresh air of heaven and earth and make life". That is to say, the sleep time at night should be delayed, but the time of getting up in the morning remains unchanged, and the nap time can be increased at noon. Autumn sleep time is to lie early and get up early, that is to say, autumn sleep time should be a little longer, in order to make up for the summer sleep hurt Yin Qi. Winter, winter master collection, should go to bed early and get up late. In the famous Ming Dynasty Qian's Xiuling Yaozhi [37], it is recorded that "lie early and rise early, keep up with the chickens, make the ambition peaceful, slow down the autumn punishment, gather the spirit, refer to the records, the lung qi is clear, the autumn Qi should be responded, and the harvest is prosperous", "early lying and late rising, warm feet and cool brain, expose the back to avoid cold, do not sweat, foot should be washed often”. Although today's people cannot sleep according to the ancient sages’ request, today's people should learn from the wisdom of ancient sages.

\subsection{Regulate emotion and improve sleep}

The main means of TCM to improve sleep and prevent sleep disorders is to gradually improve the quality of sleep, thus preventing the occurrence of sleep disorders and improving the ability to deal with sleep disorders. Zhang Zihe said in "Confucian affairs and relatives" [38]: "It is caused by thinking of Qi, which is sleepless and sleepless Recognize that emotions can affect sleep. Based on this, the author puts forward the methods of TCM Psychotherapy, such as treating fear by thinking, treating anxiety with joy over sadness, and treating insomnia with empathy and Yi Zhi. This is consistent with the modern method of improving stress and anxiety sleep through psychological counseling. Traditional Chinese medicine holds that the essence and Qi of five zang organs are the material basis of emotional activities, and emotional activities are the external manifestations of the five viscera essence. Under normal circumstances, the human body plays the function of self-regulation of emotion and defense, buffering external spiritual stimulation, and has adaptability and defense to external stimulation. However, the injury of seven emotions and excessive five emotions can lead to dysfunction of viscera function and Qi mechanism and insomnia [39]". As Zhu Danxi [40] says, "Qi and blood are in harmony, and all diseases are not born. When there is depression, all diseases are born”. This suggests that we should pay attention to the effect of mood on sleep.

\section{Syndrome differentiation and treatment of insomnia}

For example, Sun Simiao’s “Bei Ji Qian Jin Yao Fang” [40] not only discussed the relationship between deficiency and excess of viscera and sleep disorders, but also proposed Wendan Decoction, Zhuli Decoction and Fushen Decoction for treating chronic disease and gall cold, Buqi Decoction for deficiency of heart qi and Dazhen Xinsan for heart deficiency and palpitation. In addition, there are Zhenxin pills for treating "dreaminess, palpitation and loss of essence" in qianjinyi Decoction [41], dajianzhong Decoction for "communication between dreams and ghosts, loss of sperm, panic and asthenia”, and Zhenxin xingyizhi prescription and zhishuifang for sleeping more. In modern times, most doctors put forward the understanding of insomnia treatment from different angles.

\subsection{Treatment from Qi and blood}

Qi and blood are the material basis for maintaining human life activities. Qi is the commander of blood, and blood is the mother of Qi. Qi can generate blood, control blood and move blood, and blood can generate and carry Qi. Any cause of Qi dysfunction will cause qi stagnation, Qi deficiency and blood deficiency, blood stasis and other Qi and blood diseases, leading to insomnia. If the blood deficiency does not reach the extremities, the limbs are chilly and the moss is white and greasy. It is used to replenish qi and nourish blood, dispel phlegm and calm the mind. Cinnamon Twig, white peony, raw keel, raw oyster, fried jujube kernel, nocturnal vine, Yunling, chuanxiong, Zhuru, Fructus aurantii, Acorus tatarinowii, Curcuma, plantaginis, tangerine peel, 1 case a day, dizziness and palpitation were relieved ${ }^{[42]}$. In the treatment of insomnia caused by qi deficiency and blood stasis, the methods of To- 
nifying Qi and removing blood stasis, nourishing the heart and tranquilizing the mind were used. The Buyang Huanwu Decoction of Yilin Gaicuo and Jiaotai Pill of Hanshi Yitong were cut down, and the prescriptions were Shengqi, Danggui, Huanglian, cinnamon, safflower, peach kernel, Shengdi, shenglonggu, shengoyster, nocturnal Caulis, Cassia obtusifolia, Plantago, Gastrodia elata, chuanxiong, and cortex moutan [43].

\subsection{Treatment from regulating viscera}

"Spirit pivot evil guest” [44] said: "today Jue Qi guest in the five Zang Fu organs, then Wei Qi alone guard its outside, walking in Yang cannot enter into Yin, walking in Yang, Yang Qi Sheng, Yang Qiao depression, cannot enter Yin, yin deficiency, so the eyes are not dark". Therefore, the dysfunction of viscera can lead to insomnia. Zhang Qihui [45] believes that the liver is the hub of Qi and blood circulation, and the spleen and stomach are the foundation of the acquired. Therefore, he advocates that the treatment should start from regulating the liver and spleen, and the treatment should be based on soothing the liver and strengthening the spleen. Zhang Chengliang [46] thinks that the main disease of insomnia is due to lack of blood essence and Yin Yang disharmony. Therefore, Yishen Anmei decoction is often used to treat insomnia with heart kidney Disharmony Syndrome.

\subsection{Treatment from phlegm and blood stasis}

Phlegm and blood stasis can be pathological products or pathogenic factors. They can cause disease alone or simultaneously. Phlegm and blood stasis block clear the orifices, and the loss of brain collaterals can cause insomnia. Zhao Zheng [47] and others adopted Xuefu Zhuyu Decoction, which had significant effect and no adverse reactions. Cui Zhongqin [48] made the Huoxue Anshen Decoction (Danshen, Sanleng, Xiangfu, Muxiang, Danggui, amber, etc.) as the main drugs for promoting blood circulation and removing blood stasis (Danshen, Sanleng, Xiangfu, Muxiang, Danggui, amber, etc.) has achieved good clinical efficacy in the treatment of insomnia. Wang Suli [49] and other studies showed that Huanglian Wendan Decoction had a significant effect on insomnia compared with diazepam tablets alone, with an efficiency of $98.3 \%$ and no adverse reactions.

\section{Shen's understanding of Insomnia Based on syndrome differentiation and treatment}

\subsection{Discussion on syndrome differentiation and treatment of Shen's female Department insomnia}

The etiology and dialectical classification of insomnia is complex. Shen's female Division [50] classifies insomnia into two categories: Yin deficiency and fire hyperactivity and phlegm and blood stasis disturbance. Yin deficiency includes water deficiency and blood deficiency. Fire refers to heart fire and liver fire. It is characterized by less moss, red tongue, thin pulse, sweating and irritating heat. Kidney yin deficiency, liver yin deficiency and heart Yin deficiency are combined with waist and knee weakness, flank distension, palpitation; spleen yin deficiency is mainly characterized by blood deficiency, white face and limb fatigue; lung yin deficiency is accompanied by dry cough and throat dryness; patients with liver fire have red tongue and pulse string number. Liver fire hyperactivity, heart irritable, urine yellow, mouth bitter irritable, flank distended, pulse string number. Insomnia caused by phlegm and blood stasis disturbing the mind, mainly manifested as dizziness, chest tightness, stomach fullness, mouth sticky, moss turbid and greasy, and smooth pulse. There are three criteria for judging blood stasis: local blood knot syndrome, stabbing pain at fixed location, tumor refusing to press, joint deformation swelling and pain, blood stagnation syndrome of the whole body, dark face and purple lip, blue purple claw nail and dark tongue ecchymosis. And advocated the treatment of phlegm and blood stasis disturbance insomnia main medicine has three flavors: semen Raphani, Salvia miltiorrhiza, nocturnal vine.

In the treatment of insomnia, Shen's women's department put forward her own new ideas, that is, diet therapy, physical therapy and light therapy, hydrotherapy, as well as other non drug therapy combined with drug therapy.

\subsection{Shen's new thinking of insomnia treatment in female Department [50]}

\subsubsection{Diet therapy}

In terms of the influence of diet on sleep, Huangdi Neijing [51] has a saying that "if the stomach is not in harmony, then he will lie restless". The causes of "stomach disharmony and restlessness" can be summarized as two aspects: one is that the dinner is too full, the diet is too much, the stomach is congested, and the brain is relatively ischemic, resulting in difficulty in falling asleep or increasing the number of nightdreams; the other is that overeating is fat, sweet and greasy, which makes the stomach qi block. Through observation and accumulation, it is not only drug therapy that can help sleep, but also food therapy is indispensable. The foods that help sleep mainly include poria cocos, yam, lily and longan, malt, medlar, soybean milk, and auricularia, jujube, lotus seed and orange 
peel.

\subsubsection{Physical therapy}

The method of hypnosis and physical therapy is mainly indoor Taiji. When practicing Taiji, three things should be done: to relax the mind, to stabilize the state of mind, to balance the body. Don't take strenuous exercise before going to bed. Take a walk for 10 minutes as the degree, which is conducive to quiet sleep.

\subsubsection{Hydrotherapy}

There are three kinds of Spa: cold water bath, mineral bath and sea water bath.

Cold water bath can use cold water face bath and cold water foot bath. Mineral water bath is also known as hot spring bath. Its warm and thermal properties can expand capillary vessels and accelerate blood circulation. In addition, there are mechanical buoyancy and static pressure in the hot spring, which can massage and relax the human body. Mineral water contains beneficial chemical components, such as sulfur, which can eliminate fatigue, and magnesium has a sedative effect. Sea water bath also has subtle benefits to the human body. For example, salt in sea water can stimulate nerve endings through the skin, improve the blood circulation and metabolic function of the body, and have the benefits of calming and tranquilizing.

\subsubsection{Psychotherapy}

Seven emotions hurt is the main cause of insomnia, the implementation of mental therapy is the main guarantee of hypnosis, keep the mood comfortable, quiet and refreshing, can cause anger, peace of mind.

\subsubsection{Art therapy}

Active participation in noble literary and entertainment activities conducive to physical and mental health can not only cultivate sentiment, but also relax the nerves, so that the whole body and mind can be relaxed, forget the troubles, get a new self, and enter into sleep happily.

To sum up, insomnia belongs to "insomnia" in traditional Chinese medicine, with different appellations in different dynasties. There are many causes of the disease. It is generally believed that emotional disorders, improper diet, fatigue and physical deficiency are the causes of the disease. In the treatment of insomnia, most experts proposed that the treatment of deficiency and excess of Zang Fu organs, or qi, blood, phlegm and blood stasis had achieved ideal curative effect [52]. However, there are still some deficiencies in the treatment of insomnia in traditional Chinese medicine. There is still a lack of strict and unified standard for the diagnosis and efficacy of insomnia, and there is a lack of objective judgment of clinical efficacy. There is a lack of experimental and other basic research to clarify the mechanism of insomnia. Therefore, based on years of clinical experience, Shen's Department of gynecology collected a large number of insomnia patients' medical records, summarized and innovated the new idea of "comprehensive application of hypnosis", which made TCM more perfect in the treatment of diseases, and was favored and recognized by clinicians. In short, traditional Chinese medicine treatment of insomnia is a reliable method, broad prospects, worthy of promotion.

\section{References}

[1] Yang Mingzhu. (2019). Exploring the distribution of syndrome elements of insomnia with depression and anxiety [D]. Beijing University of traditional Chinese medicine.

[2] Wang Haiyan, Li Siyi, Zhang Yi, Peng Wei. (2020). Systematic review of Integrated Chinese and Western medicine in the treatment of insomnia [J]. Shandong Journal of traditional Chinese medicine, 2020, 02: 134-139.

[3] Zhou Zhongying. (2012). Internal medicine of traditional Chinese medicine, 9th Edition [M]. Beijing: China traditional Chinese Medicine Press, 2012: 149.

[4] Ouyang 84. (2016). A comparative study on the meridians circulation of "foot and arm ten one pulse moxibustion classic" and "Yin Yang ten one pulse moxibustion Jing” [J]. Chinese medicine information, 2016, 05: 98-101.

[5] Liang Jiankang, Cheng Linbi, Liang Jun, Zhao Boyuan, Huang Chang, Zhang Qicheng. (2019). An analysis of the theory of health preservation and its ideological origin in Mawangdui Jian Shu Shi Wen [J]. Journal of Liaoning University of traditional Chinese Medicine, 2019, 08: 73-75.

[6] Wei Shixiong, Liu Qiong, Wang Ping. (2018). Discussion on insomnia caused by five zang organs in Treatise on Febrile Diseases and treatment from theism [J]. Journal of Traditional Chinese Medicine, 2018, 59(13): 1169-1170.

[7] (Eastern Han Dynasty) Zhang Zhongjing. (2007). Treatise on febrile diseases [M]. Beijing: China traditional Chinese Medicine Press.

[8] (Eastern Han Dynasty) Zhang Zhongjing. (2007). Synopsis of golden chamber [M]. Beijing: China traditional Chinese Medi- 
cine Press.

[9] Wang Xudong. (2017). Wang Shuhe and the historical facts of pulse meridian revisited [J]. Chinese Journal of traditional Chinese medicine, 2017, 10: 4364-4366.

[10] Wang Tao. (2007). External Taiwan secret collection [M]. Shenyang: Liaoning science and Technology Press, $2007: 340$.

[11] Huang, Wan Yuan. (2014). Zhongjing's theory of syndrome differentiation and treatment of insomnia and its clinical application research [D]. Nanjing University of Traditional Chinese Medicine.

[12] Chen Yan (Wu Xuan), Southern Song Dynasty. (2007). On prescriptions of three causes and extreme diseases [M]. Beijing: China Traditional Chinese Medicine Science and Technology Press.

[13] Liu Yuxin, Liu Xiaoyan. (2016). Literature research on syndrome differentiation and treatment of insomnia in song, Jin and Yuan Dynasties [J]. Journal of Guangzhou University of Traditional Chinese Medicine, 2016, 01: 141-146.

[14] Dong Xu. (2018). Literature research on TCM etiology and pathogenesis of insomnia in Ming and Qing Dynasties [D]. Changchun University of Traditional Chinese Medicine.

[15] Social and historical background of medical development in song, Jin and Yuan Dynasties [J]. Tang Weihua. Journal of Liaoning University of traditional Chinese medicine. 2008 (07).

[16] Liu Yuxin, Liu Xiaoyan. (2017). Syndrome differentiation and treatment of insomnia in Ming and Qing Dynasties [J]. Shanghai Journal of Traditional Chinese Medicine, 2017, 08: 43-46.

[17] Ling Yan, Liu Shulin. (2015). Research on TCM disease name of insomnia [J]. Acta Sinica Sinica, 2015, 211(30): 1848.

[18] Ma Dandan, Wang Linlin. (2020). Research progress of traditional Chinese medicine in the treatment of insomnia [J]. Chinese Folk Therapy, 2020,04: 98-100

[19] Wei Gang, Wei Ting. (2019). Research progress on TCM syndrome differentiation and treatment of insomnia [J]. World Latest Medical Information Digest, 2019, 18: 44-45.

[20] Jiang Suhua, Gao Xin. (2019). Research progress of emotional nursing combined with traditional Chinese medicine in the treatment of insomnia [J]. Feet and Health Care, 2019, 14: 5-6.

[21] Zhang Hong. (2019). Analysis of the treatment of blood syndrome from spleen [J]. Journal of Hunan University of Traditional Chinese Medicine, 2019, 07: 870-873.

[22] Wang qiechu. (2006). TCM diagnosis, differentiation and treatment of insomnia [J]. Bulletin of Traditional Chinese Medicine, 2006, 12(5): 5.

[23] Yuan yunshuo. (2008). Treatment of 56 cases of insomnia of liver fire disturbing heart type with Longdan Xiegan Decoction [J]. Journal of Liaoning University of Traditional Chinese Medicine, 2008(8): 107-108.

[24] Duan Yongqiang, Ma Jun, Yang Xiaoyi, Li Nenglian, Wang Huinan, Mou Dehai, Gong Zihan, Bai min. (2020). Examples of modified Sijunzi Decoction in treating vertigo from the perspective of spleen governing Shengqing [J]. Chinese Medicine Research, 2020, 04: 40-41.

[25] Su Wen. (2011). [M]. Beijing: China Medical Science and Technology Press, 2011.

[26] Yuan Dongchao, Yang Mingqian, Ni Fei, Chen Yanjun, Dong Ye, Wang Caixia, Cui Jiapeng. (2020). On the teaching concept of ontology application in Neijing [J]. Modern Distance Education of Chinese Medicine, 2020, 02: 3-5.

[27] Shen Hao, Liu Hongquan. (2019). Analysis on the treatment of refractory insomnia from spleen [J]. Journal of Modern Integrated Chinese and Western medicine, 2019, 21: 2364-2366 +2394.

[28] Zhang Min, Ji Lijin, Huang Junshan. (2016). From the perspective of spleen depression, insomnia is treated from the middle Jiao [J]. Chinese Journal of Traditional Chinese Medicine. 2016(10).

[29] Chen Chen, Wang Peng, Xie Huanhuan. (2016). An analysis of the treatment of insomnia by Lin Peiqin in Lei Zheng Zhi Cai [J]. Journal of Jiangxi University of Traditional Chinese Medicine, 2016, 05: 15-16.

[30] Professor Cao Jiani. (2016). Peiyuan's experience in treating insomnia from spleen and stomach [D]. Liaoning University of Traditional Chinese Medicine.

[31] Sun Qingchun, Wang Enlong. (2013). Etiology and pathogenesis of insomnia [J]. Journal of Practical Chinese Medicine, 2013, 22: 83-85.

[32] Zhang Jie. (2019). Research on diagnosis and treatment of psychosomatic diseases in xumingyilei case [D]. Yunnan University of traditional Chinese medicine.

[33] Bao Jianmin, LV Ping, Chen Zhenping, Mou Chonglin. (2020). On the core idea of Liu Hejian's “theory of fire and heat” and its influence on the formation of Jin and Yuan four masters [J]. New Chinese medicine, 2020, 06: 178-181.

[34] Jiang Zengwei, Xu Xiaomei. (2020). Discussion on Li Dongyuan’s pulse method and "Yin Fire” theory [J]. Chinese Medicine Guide, 2020, 03: 103-105.

[35] Zhang Qingsong, Yin Jianping, Li Yingzhen, Huang Liansha, Chen Baishu, Cao Yuan, Wang Yingyun. (2018). Research Progress on correlation between Sub-health insomnia and meridians [J]. Clinical Research of Traditional Chinese Medicine. 
2018(04).

[36] Ou Yangjun. (2019). Laozi’s health culture (I) [J]. Health Monthly, 2019, 08: 676-680.

[37] Sha Tao. (2004). Explanation of “Xiuling Yaozhi” by Leng Qian [a]. Youth Working Committee of Chinese Association of Integrated Chinese and Western medicine. Proceedings of the Fifth National Symposium on the integration of Chinese and Western Medicine [C]. Youth Working Committee of Chinese Association of Integrated Chinese and Western medicine: 2004: 1.

[38] Gao Nannan, Yi Bowen, Chen Zijie, Wu Yufeng. (2019). Analysis of emesis in Confucian School of affairs [J]. Journal of Beijing University of Traditional Chinese Medicine, 2019, 07: 546-548.

[39] Liu Bing. (2019). Theoretical and data mining research on the treatment of insomnia from "Five Spirits and viscera” [D]. Liaoning University of Traditional Chinese Medicine.

[40] Ji Xiaoyu, Tao Yingli. (2020). Ye ping.zhu Danxi’s academic thought and the relationship between medication and regional characteristics [J]. Zhejiang Journal of integrated traditional Chinese and Western medicine, 2020, 01:83-84.

[41] Yao Fengyun, Luo Huafu, Wang Bingzhi. (2017). Study on the compatibility law of spleen system prescriptions in Bei Ji Qian Jin Yao Fang by Sun Simiao [J]. Shi Zhen traditional Chinese medicine and Chinese medicine, 2017, 06: 1533-1535.

[42] Chen Yingqun, Chen Yi, Li Taotao, Lin Yixin, Zhang Min, Chu Yongqing, Yan Xin. (2019). Chinese medicine master Yan Dexin's academic thoughts on treating insomnia from Qi and blood [J]. Shanghai Journal of Traditional Chinese Medicine, 2019, 03: 1-4.

[43] Han Xuejie, Li Chengwei. (2006). Selected cases of Shen Shaogong [M]. Beijing; Xueyuan Publishing House. 2006.

[44] Lingshu (1956). [M]. Beijing: People’s Health Publishing House, 1956.

[45] Zhang Qihui. (2005). Treating insomnia from regulating liver and spleen [J]. Sichuan Traditional Chinese medicine, 2005, 23(12): 9-11.

[46] Zhang Chengliang. (2012). Treatment of 48 cases of insomnia with Yishen Anmei Decoction [J]. Guangming Traditional Chinese Medicine, 2012, 27 (7): 1360.

[47] Huang Chunhui, Li Qiyi. (2018). Professor Li Qiyi's clinical experience in treating insomnia from phlegm and blood stasis [J]. Journal of Zhejiang University of Traditional Chinese Medicine, 2018, 03: 190-192.

[48] Cui Zhongqin. (2006). Curative effect observation of self-made Huoxue Anshen Decoction on 124 cases of insomnia [J]. Sichuan Traditional Chinese medicine, 2006, 24 (10): 55-56.

[49] Wang Suli, Zhang Ming. (2006). Treatment of 86 cases of insomnia with Huanglian Wendan Decoction [J]. Hunan Traditional Chinese Medicine, 2006, 22 (4): 42.

[50] Shen Shaogong. (2004). General strategy of traditional Chinese medicine [M]. Beijing: Science Press, 2004.

[51] Shao Xiangyun, Zhao Min, Li Dan. (2018). Analysis of insomnia treatment based on "Huangdi Neijing” and law theory [J]. Clinical Research of Traditional Chinese Medicine, 2018, 33: 20-22.

[52] Wang Yanhua. Professor Zheng Shaozhou's experience in treating insomnia from viscera [J]. Guangming TCM, 2017, 02: 176-177. Zhi, 2019, 12: 963-964 + 969. 